\title{
3 Die juristische Aufarbeitung der Kampagne gegen Rechtsabweichler
}

Hinsichtlich des Verständnisses über den konkurrierenden Diskurs um die Kampagne gegen Rechtsabweichler sollte zunächst die juristische Aufarbeitung der Einzelfälle einer genaueren Betrachtung unterworfen werden. Gemeint ist damit die formale Wiedereingliederung der Rechtsabweichler in die Gesellschaft sowie die finanzielle Kompensation der Opfer. Der juristische Umgang mit den Opfern der Kampagne gegen Rechtsabweichler spielt auch eine entscheidende Rolle im Kampf um Chinas kollektives Gedächtnis. Denn die politischen Entscheidungen rund um die juristische Behandlung der Opfer durch den Staat und die daran geäußerte Kritik wurden auch zu einem wichtigen Bestandteil der inoffiziellen Geschichtsschreibung - sowohl innerhalb der staatlich geduldeten als auch innerhalb der in der VR China zensierten. Wie in den folgenden Abschnitten erläutert, brachte eine Reihe jener Intellektueller, die sich nach dem Tode Maos an einer alternativen Geschichtsschreibung beteiligten, neben ihrer Auseinandersetzung mit dem Erlebten innerhalb des Laogai-Systems, immer wieder zum Ausdruck, dass sie sich als Opfer von der Partei nach dem Tode Maos juristisch nicht gerecht behandelt fühlten. Das heißt die Art des kritischen, öffentlichen Diskurses wurde Jahrzehnte nach dem Ende der Kampagne auch durch die grundlegenden Entscheidungen bezüglich des juristischen Umgangs mit den Unschuldigen ab den späten 1970er Jahren geprägt. Dadurch wurden Forderungen nach angemessenen Kompensationszahlungen für die Opfer, neben weiteren Forderungen wie etwa der nach einer offiziellen Neudeutung der Geschichte, im Laufe der Zeit auch zum Bestandteil einer alternativen Geschichtsschreibung. Dies wird auch immer wieder in jenen Memoiren oder Kurzmemoiren der Opfer deutlich, in denen sich die Autoren neben der Kampagne an sich auch kritisch mit der staatlichen Ungerechtigkeit gegenüber den ehemaligen Rechtsabweichler auseinandersetzen.

Generell sollte man aber nicht davon ausgehen, dass eine umfassende juristische Aufarbeitung eine weitere Beschäftigung in Form von Erinnerungen ausschließen kann bzw. dass dies einen kritischen öffentlichen Diskurs in Gänze verhindert, wie man anhand zahlreicher globalhistorischer Beispiele sehen kann. Zum Beispiel schloss sich an die umfassende juristische Aufarbeitung des Holocaust, die nicht nur mit einer finanziellen Entschädigung der Opfer, sondern auch mit einer Anklage von Tätern einherging, allmählich ein Prozess des individuellen

Ә OpenAccess. ( 2021 Anja Blanke, publiziert von De Gruyter. (cc))BY-NC-ND Dieses Werk ist lizenziert unter der Creative Commons Attribution-NonCommercial-NoDerivatives 4.0 International Lizenz.

https://doi.org/10.1515/9783110719253-005 
und kollektiven Erinnerns an. ${ }^{120}$ In den 1980er Jahren entbrannte zudem eine kritische Debatte über den juristischen Umgang mit den „vergessenen Opfern“, die als ausländische Zwangsarbeiter in Kriegsgefangenschaft waren und keine Entschädigungszahlungen erhalten hatten. ${ }^{121}$

Außerdem lässt sich generell die Frage danach, welche Kompensation für ein Opfer eines historischen Verbrechens oder im chinesischen Kontext eines „historischen Fehlers“ überhaupt „angemessen“ ist, nur schwerlich beantworten. Insgesamt macht die juristische Aufarbeitung immer nur einen Teil des Aufarbeitungsprozesses eines historischen Ereignisses aus. Dies gilt insbesondere auch für die Opfer der Kampagne gegen Rechtsabweichler.

\subsection{Rehabilitierungen während der Mao-Ära}

Bis zum Ende der Kulturrevolution war es für die Rechtsabweichler grundsätzlich sehr schwierig, eine vorzeitige Entlassung aus dem Laogai-System bzw. eine Rehabilitierung $\mathrm{zu}$ erwirken. Schließlich stellten die darin getätigten Umerziehungsmaßnahmen einen wichtigen Teil in Maos Kampf gegen die Feinde des Sozialismus dar. Eine vorzeitige Entlassung der Mehrzahl der Rechtsabweichler aus dem System hätte möglicherweise nach außen hin das Bild vermittelt, dass Mao und seine Anhänger sich auf dem politisch falschen Wege befunden haben.

Ob ein Rechtsabweichler überhaupt eine Chance hatte, nach nur wenigen Jahren aus dem Laogai-System wieder in die Gesellschaft entlassen zu werden, hing nicht nur damit zusammen, ob dieser sich erfolgreich „transformiert“ hatte, sondern zunächst damit, ob man durch die Partei als Feind des Volkes oder noch immer als ein Teil des Volkes eingestuft worden war. Entsprechend wurden die sogenannten Rechtsabweichler auch innerhalb des Systems der chinesischen Zwangsarbeitslager entweder in einem sogenannten Laogai 劳改 (für die Feinde des Systems) oder einem Laojiao 劳教 (jene, die noch als Teil der Gesellschaft angesehen wurden, aber „umerzogen“ werden mussten) $)^{122}$ untergebracht. Während Erstere als Kriminelle eingestuft worden waren und auch keinen Lohn erhielten, war es hingegen vorgesehen, Letztere durch die Umerziehung durch Ar-

120 Dieser Prozess war vor allem in der frühen Bundesrepublik durch „soziokulturelle Erinnerungskonflikte“ geprägt. Siehe Bauerkämper 2012, S. $295 \mathrm{ff}$.

121 Vgl. Hockerts 2013, https://www.bpb.de/apuz/162883/wiedergutmachung-in-deutschland19451990-ein-ueberblick?p=1.

122 Der Begriff Laogai-System hat sich in den letzten Jahren für das gesamte System der Zwangsarbeitslager in China als Oberbegriff durchgesetzt und wird in dieser Arbeit auch entsprechend verwendet. 
beit irgendwann wieder in die Gesellschaft zu integrieren. Sie erhielten während ihres Arrests auch einen Lohn für ihre Arbeit, auch wenn dieser in der Regel deutlich geringer ausgefallen ist als der Lohn für vergleichbare Arbeit außerhalb des Laogai-Systems. ${ }^{123}$

$\mathrm{Zu}$ Beginn der Kampagne gegen Rechtsabweichler war es noch vorgesehen gewesen, einen großen Teil jener, die den „Hut des Rechtsabweichlers“ trugen, bereits nach nur wenigen Jahren wieder in die Gesellschaft zu entlassen. Einige Memoiren von ehemaligen Rechtsabweichlern lassen erkennen, dass viele von ihnen dementsprechend zunächst davon ausgegangen waren, nach wenigen Monaten oder Jahren wieder in Freiheit zu sein. Die wenigsten von ihnen dürften geahnt haben, dass die meisten von ihnen, wenn sie es denn überlebten, rund zwanzig Jahre im System der Zwangsarbeitslager verbringen würden. So berichtete der später als Dissident bekannt gewordene, ehemalige Rechtsabweichler Harry Wu, dass der Polizist, der ihn im Jahr 1958 in das Zwangsarbeitslager überführte, ihm mit auf den Weg gegeben habe: „Eigentlich ist die Umerziehung durch Arbeit gar nicht mal so schlecht; du bist noch jung, es wird gut für dich sein, ein wenig zu arbeiten! Du arbeitest ein paar Monate und dann kommst du wieder zurück!" 124 Es war wohl die Hoffnung auf eine baldige Rückkehr in die Gesellschaft, die viele Rechtsabweichler in jenen Jahren zunächst angetrieben hatte, sich den Umerziehungsmaßnahmen und der damit verbundenen harten Arbeit zu beugen, um so möglichst schnell demonstrieren zu können, dass sie zu einem „neuen sozialistischen Menschen“ geworden waren. Allerdings, so berichtet es auch Harry Wu, bestand das Problem darin, dass es keine eindeutigen Kriterien und keine festgelegte Zeitdauer für eben jene Verbesserung hin zu einem ,guten, sozialistischen Menschen“ gegeben habe. Und so fragte er sich damals: „Was zeichnete eine ,erfolgreiche Verbesserung‘ eigentlich aus? Welche Maßstäbe wurden dabei angelegt? Denn als ich ins Umerziehungslager kam, sagte man mir: ,Ihr seid der Abschaum der Gesellschaft. Das wird jetzt eure Beschäftigung sein. ${ }^{\prime \prime 125}$

Tatsächlich wurde eine erste Initiative zur Entfernung des „Rechtsabweichlerhutes“ jedoch bereits im September 1959 gestartet. Im Zuge dieser Initiative wurden bis zum Frühjahr 1960 landesweit rund 8,5 Prozent aller Rechtsabweichler rehabilitiert. Voraussetzung hierfür war die vollständige Umerziehung des jeweiligen Rechtsabweichlers. Es gab allerdings kaum Transparenz darüber, welche Kriterien tatsächlich für die Entlassung der Betroffenen angewendet

$123 \mathrm{Zu}$ den unterschiedlichen Arten der Zwangsarbeitslager siehe Mühlhahn 2009.

$124 \mathrm{Wu}$ 2009, S. 76.

125 Ebd., S. 78. 
wurden. Ning Wang geht davon aus, dass die aus dem Großen Sprung resultierende, einsetzende Hungersnot einen großen Einfluss auf die zu jenem Zeitpunkt recht große Anzahl an Entlassungen der Rechtsabweichler aus den Umerziehungslagern gespielt haben dürfte. ${ }^{126}$ Die Formulierung im Beschluss vom 17. September 1959 blieb äußerst vage und besagte lediglich, dass jenen der Hut abgenommen werden sollte, die sich im Denken und Handeln von „gut zu böse“ verändert hätten:

Anyone who has changed from evil to good and has shown that he has really changed in both views and activities will no longer be considered a bourgeois rightist from now on, that is, the label of "rightist" shall be removed from him. The unit in which he works should draw a conclusion according to his accomplishments in work and study and announce it to the public. $^{127}$

Im Oktober 1959 empfing Mao eine polnische Delegation in Peking. Im Zuge eines Gesprächs mit dem Delegationsmitglied Alexander Zawadzki betonte er, dass man rund 450.000 sogenannte Rechtsabweichler enttarnt und entsprechend bestraft habe und er davon ausgehe, in den kommenden sieben bis acht Jahren jährlich 10 Prozent der Rechtsabweichler durch Umerziehung zurück in die Gesellschaft entlassen zu können. Nach Maos eigener Einschätzung des Jahres 1959 sei es demnach möglich, rund 80 Prozent von ihnen umzuerziehen - bei 10 bis 20 Prozent der Rechtsabweichler sei eine Umerziehung seiner damaligen Einschätzung nach wahrscheinlich nicht möglich. ${ }^{128}$

Unterstützung erhielten die Opfer der Kampagne bereits in den späten 1950er Jahren durch Hu Yaobang 胡耀邦, zwischen 1952 und 1966 Vorsitzender der Kommunistischen Jugendliga, was zu jener Zeit sehr ungewöhnlich war. Und so dürfte er in jenen Jahren einer von nur sehr wenigen Vertretern hoher Parteikreise gewesen sein, der sich so stark für die Interessen der Rechtsabweichler einsetzte. Er äußerte während der Jahre 1957 bis 1959 immer wieder Kritik an der Umsetzung der Kampagne und am Umgang mit den Opfern. Außerdem unternahm er immer wieder Versuche - mal mehr, mal weniger erfolgreich -, Widerstand gegen die Verurteilung einzelner zu leisten. Seine politische Haltung im Umgang mit den sogenannten Rechtsabweichlern sollte insgesamt auch einen entscheidenden Einfluss auf den Umgang mit Opfern anderer politischer Kampagnen während der Jahre nach dem Tode Maos nehmen. ${ }^{129}$

126 Wang 2016, S. 163.

127 Decision of the CCP Central Committee and the State Council concerning rightists who have shown that they have been really corrected. Union Research Institute 1971, S. 338.

128 Record of Conversation between Polish Delegation and PRC Leader Mao Zedong 14.10.1959.

129 Goldman 1991, S. 220 f. 
Weitere vereinzelte „Entfernungen von Hüten“ gab es zu Beginn der 1960er Jahre immer wieder - auch hier meist mit nur geringer Transparenz hinsichtlich der Kriterien. Beispielsweise ist den Akten des ehemaligen Rechtsabweichlers Sun Chunqi 孙春骑 zu entnehmen, dass sein Hut am 26. Januar 1961 nach drei Jahren und drei Monaten der Umerziehung entfernt worden ist. Er war einer von insgesamt 109 Rechtsabweichlern, die im Zuge dessen wenige Tage später aus einem Umerziehungslager in Luhua 芦花 (Provinz Sichuan) entlassen worden sind. Sun, der zu Beginn der Kampagne gegen Rechtsabweichler als Angestellter eines LKW-Werks in der Region Ningxia 宁夏, einem autonomen Gebiet im Nordwesten Chinas, gearbeitet hatte, war am 05. August 1958 aufgrund seiner angeblich schlechten Arbeitsleistung, einer Beteiligung an einem Streit in seinem privaten Umfeld sowie aufgrund rechtsabweichlerischer Äußerungen, die er in seinem beruflichen Umfeld getätigt haben soll, welche in seinen Akten jedoch nicht genauer spezifiziert werden, als Rechtsabweichler verurteilt und am 09. Oktober 1958 inhaftiert worden. Im Jahr 1978 legte er rund 17 Jahre nach seiner Entlassung aus dem Laogai-System schließlich mehrfach schriftlich Beschwerde bei der Abteilung für öffentliche Sicherheit ein, um die genauen Gründe für seine damals auf drei Jahre angesetzte Umerziehung zu erfahren. In seinen Beschwerdeschreiben beteuert er, dass er sich nie negativ über die Partei oder Mao geäußert habe, weshalb er die Gründe für seine Verurteilung auch rund 20 Jahre danach noch immer nicht nachvollziehen könne. Den Briefen ist ebenfalls zu entnehmen, dass die Gründe für die Entlassung ebenso unklar waren wie die Gründe seiner Verurteilung. ${ }^{130}$ Es ist durchaus denkbar, dass Sun Chunqis Schicksal stellvertretend für das vieler Rechtsabweichler steht, nämlich, dass er nur deshalb verurteilt worden war, weil die von oben ausgegebenen Quoten zur Enttarnung von Rechtsabweichlern oder Konterrevolutionären eingehalten werden mussten, und nicht, weil er sich tatsächlich eines Verbrechens schuldig gemacht hatte. Es ist auf der anderen Seite ebenso möglich, dass man insbesondere diejenigen im Jahr 1961 für die Entfernung der Hüte in Betracht zog, die aus besonders nichtigen Gründen verurteilt worden waren. ${ }^{131}$

Während der 7000-Kader-Konferenz, welche zwischen dem 11. Januar und dem 07. Februar 1962 stattfand und dem Zweck dienen sollte, die politischen Er-

130 Communist Chinese Political Movement Collection, 2014 C24.

131 Das Quotensystem der Kampagnen der 1950er Jahre geht auf Maos Annahme zurück, dass rund 10 Prozent der Bevölkerung politisch abweichlerische Tendenzen aufweisen würden. Für die Enttarnung der Rechtsabweichler wurde, je nach Arbeitseinheit, eine Quote zwischen 1 und 3 Prozent bzw. 5 und 10 Prozent festgelegt. Die Kriterien für die Festlegung der Höhe der Quoten waren nicht transparent. In einigen Arbeitseinheiten wurde sogar eine Quote von bis zu 23 Prozent erreicht. Wang 2016, S. 39 f. 
fahrungen der vergangenen zwölf Jahre zu analysieren, insbesondere aber die Fehler des Großen Sprungs nach vorn, wurden auch die Rehabilitierungen von Parteikadern, Parteimitgliedern und gewöhnlichen Bürgern thematisiert, die in den Jahren zuvor zu Unrecht als Rechtsabweichler gebrandmarkt worden waren. Im Ergebnis wurden vereinfachte Verfahren zur schnelleren Rehabilitierung der Rechtsabweichler beschlossen. ${ }^{132}$ Mao selbst rechtfertigte während der Konferenz in seiner Rede vom 30. Januar 1962 jedoch sein politisches Handeln gegen die „Feinde des Volkes“, welche seiner Einschätzung nach 4 bis 5 Prozent der Gesamtbevölkerung ausmachten und deshalb zur Reform gezwungen werden müssten:

Die demokratische Diktatur des Volkes unterdrückt die Grundherren, Großbauern, Konterrevolutionäre, die üblen Elemente und antikommunistischen Rechten. Die Konterrevolutionäre, die üblen Elemente und antikommunistischen Rechten repräsentieren die Grundherrenklasse und die reaktionäre Bourgeoisie. Diese Klassen und schlechten Menschen machen etwa vier bis fünf Prozent der Gesamtbevölkerung aus. Solche Leute müssen wir zur Umerziehung zwingen. Sie sind Objekt der demokratischen Diktatur des Volkes. Auf wessen Seite stehen wir? Auf Seiten der Volksmassen, d.h. von über 95 Prozent der Gesamtbevölkerung, oder auf seiten der Grundherren, Großbauern, Konterrevolutionäre, üblen Elementen und Rechten, die nur vier bis fünf Prozent der Bevölkerung ausmachen? Wir müssen auf der Seite der Volksmassen stehen, auf keinen Fall dürfen wir auf der Seite der Feinde des Volkes stehen. Für Marxisten-Leninisten ist dies eine Frage des grundlegenden Standpunkts. ${ }^{133}$

Dabei sprach er allerdings auch über die Notwendigkeit, jene Kader, die fälschlich zu Feinden des Volkes verurteilt worden waren, zu rehabilitieren. Gleichzeitig unternahm er den Versuch, den positiven Aspekt in der falschen Behandlung der Kader zu unterstreichen, indem er auf die Möglichkeit hinwies, dass diese dadurch fortan die Massenlinie besser umsetzen könnten:

Auch wir haben in der Vergangenheit einige Kader falsch behandelt. Wurden sie völlig oder auch nur teilweise falsch behandelt, so müssen die Fälle den konkreten Umständen entsprechend überprüft und korrigiert werden. Aber allgemein gesagt, stählt solche falsche Behandlung - Degradierung oder Versetzung - ihren revolutionären Willen und ermöglicht ihnen zudem, aus den Volksmassen neue Erkenntnisse zu schöpfen. ${ }^{134}$

Tatsächlich fiel die Zahl der Rehabilitierungen von Rechtsabweichlern der Jahre 1959 bis 1976 insgesamt jedoch recht niedrig aus und so sollten Entlassungen aus

132 MacFarquhar 1997, S. 179.

133 Mao 1978, S. 16 Die (überarbeitete) Rede wurde am 01. Juli 1978 in der Renmin Ribao veröffentlicht.

134 Ebd., S. 5. 
dem Laogai-System vor dem Tode Maos eher die Ausnahme bleiben. Insbesondere während der Kulturrevolution wurden nur sehr wenige Rechtsabweichler freigelassen, vor allem deswegen, weil sich der Ton in Bezug auf den Umgang mit Klassenfeinden, politischen Abweichlern respektive Konterrevolutionären verschärft hatte, weswegen auch Entlassungen von Rechtsabweichlern noch schwieriger zu legitimieren gewesen sein dürften. Die gesellschaftliche Stimmung während der Kulturrevolution richtete sich gegen jene, die der Diktatur des Proletariats (potentiell) entgegenstanden, und dazu kann man die Rechtsabweichler, aus Sicht der damaligen Revolutionäre, zweifelsohne hinzuzählen. Die rechtliche Situation hatte sich für die „Feinde der Diktatur des Proletariats“ ohnehin bereits verschlechtert, da Artikel 85 der 1954er-Verfassung („Alle Bürger der Volksrepublik China sind vor dem Gesetz gleich.“)135 bereits zu Beginn der Kampagne gegen Rechtsabweichler scharf kritisiert worden war und für Konterrevolutionäre und Rechtsabweichler faktisch nicht mehr galt - mit der zweiten Verfassung, welche 1975 verabschiedet worden war, wurde Artikel 85 schließlich gänzlich abgeschafft. ${ }^{136}$ Bedacht werden muss auch, dass sich die Durchsetzung des Rechts während der Kulturrevolution insgesamt sehr stark verändert hatte. Das chinesische Rechtssystem im China unter Mao operierte innerhalb der Grenzen politischer Restriktionen und so funktionierte es während der Kulturrevolution noch stärker als zuvor in Einklang mit den damaligen „revolutionären Theorien“. Konkret bedeutete dies, dass es dem Prinzip der „Diktatur der Massen“ entsprach und in seiner Rechtsprechung der „Massenlinie“ folgte - meist hatten öffentliche Diskussionen über den jeweiligen Fall und Anhörungen über die Verfehlungen des Konterrevolutionärs oder des Rechtsabweichlers stattgefunden, was wiederum starken Einfluss auf das Urteil hatte. Die Rechtsordnung beugte sich in jenen Jahren dem Prinzip der „Diktatur über den Feind und den Schutz der revolutionären Massen“. ${ }^{137}$

Aufgrund der gesellschaftlichen Stimmung seit dem Beginn der Kulturrevolution, gestaltete sich die Rückkehr in die Gesellschaft für jene, die dennoch vorzeitig entlassen worden waren, meist äußerst schwierig. In den Augen der Roten Garden hatten sie den Stempel „Rechtsabweichler“ nicht unbedingt verloren und deshalb mussten sie und ihre Angehörigen in der Regel auch weiterhin mit Diskriminierungen rechnen.

135 Verfassung der Volksrepublik China (Erste Mao-Verfassung) vom 20. September 1954.

136 Verfassung der Volksrepublik China (Zweite Mao-Verfassung), angenommen auf der Ersten Tagung des IV. Nationalen Volkskongresses am 17. Januar 1975, 1975.

$137 \mathrm{Xu}$ 2018, S. $27 \mathrm{ff}$. 


\subsection{Einzelfallkorrekturen nach dem Tode Maos}

Wie bereits erwähnt, hatte $\mathrm{Hu}$ Yaobang einen entscheidenden Einfluss auf die Rehabilitierungen von Intellektuellen. Nach dem Tode Maos im September 1976 schloss er sich Deng Xiaopings Rückkehr an die Macht an und wurde bereits im März 1977 zum Vizepräsidenten der Zentralen Parteischule der KPCh. Im August 1977 wurde er schließlich zum Mitglied des Zentralkomitees gewählt. Alsbald begann er, seine hohe Position dafür zu nutzen, die Entlassungswelle tausender während der Kampagne gegen Rechtsabweichler und der Kulturrevolution zu Unrecht Verurteilter in Gang zu setzen. Deng Xiaoping zeigte sich in Bezug auf die Entlassungen von Rechtsabweichlern zunächst noch recht zögerlich und wollte zum damaligen Zeitpunkt nur rund 60 Prozent der Rechtsabweichler und Konterrevolutionäre rehabilitieren. ${ }^{138}$ Entscheidend für Dengs Zögern dürfte auch seine Rolle während der Kampagne gegen Rechtsabweichler gewesen sein, da er diese als Generalsekretär des Zentralkomitees entscheidend umgesetzt hatte. Ihm dürfte gewahr gewesen sein, dass insbesondere in einer Phase des Umbruchs eine Debatte über seine Beteiligung an der Kampagne ein Problem für seine Machtbestrebungen darstellen könnte. ${ }^{139} \mathrm{Hu}$ Yaobang übte großen Einfluss auf die sogenannte Theorie-Konferenz der KPCh (Lilun gongzuo wuxu hui 理论工作务虚会) aus, welche zu Beginn des Jahres 1979 abgehalten wurde und zum Ziel hatte, eine ideologische Einigkeit zwischen den Intellektuellen zu erreichen. Unterstützt wurde er vom Propagandaministerium und der Chinesischen Akademie der Sozialwissenschaften. Zuspruch erhielt Hu Yaobang in diesem Zusammenhang damals vor allem von Yu Guangyuan 于光遠, der seit 1975 ein hochrangiges Mitglied der Zentralen Forschungsstelle der KPCh war und der im Zuge dessen Fehler, die während der Kampagne gegen Rechtsabweichler, des Großen Sprungs nach vorn und während der Kulturrevolution durch die Partei begangen worden waren, kritisierte. ${ }^{140}$ Yu forderte dabei auch die Einschränkung der Staatsmacht und die Stärkung der Macht des Volkes, da „historische Fehler“ wie die Kampagne gegen Rechtsabweichler seiner Einschätzung nach eine direkte Folge der Parteiendiktatur gewesen seien. ${ }^{141}$

Offiziell wurde bereits mit den Beschlüssen zur „Entfernung der Hüte“, welche einige Monate vor der Konferenz im April und September des Jahres 1978 vom Zentralkomitee herausgegeben worden waren, mit der juristischen Überprüfung

138 Goldman 1991, S. 222.

139 Zur Rolle Deng Xiaopings während der Kampagne gegen Rechtsabweichler siehe Chung 2011.

140 Sleeboom-Faulkner 2007, S. 59f.

141 Ding 1994, S. $91 \mathrm{ff}$. 
und der Freilassung hunderttausender zu Unrecht als Rechtsabweichler verurteilter Intellektueller begonnen. ${ }^{142}$ Das Dokument Nr. 11 vom 05. April 1978 besagt, dass die Hüte aller Rechtsabweichler zu entfernen seien, sie fortan nicht mehr als solche bezeichnet werden dürften, dass sie aus den Arbeitslagern entlassen werden sollten und dass auch die Hüte der bereits verstorbenen Rechtsabweichler posthum entfernt werden sollten. Weiterhin besagt das Dokument, dass die Kinder der Rechtsabweichler keine gesellschaftliche Diskriminierung mehr erfahren dürften. Das heißt, dass sie fortan aufgrund dessen auch keine Nachteile mehr bei der Entscheidung über die Zuteilung eines Studienplatzes oder über die Aufnahme in die Partei erleiden sollten. Weiterhin sprach das Dokument den Rechtsabweichlern das Recht zu, eine Arbeitsstelle zugeteilt zu bekommen, die ihrer ursprünglich erlangten Qualifikation entsprach. Ein weiterer wichtiger Beschluss des Dokuments war die Regelung zur Umsiedlung der Rechtsabweichler, die seit ihrer Verurteilung zur Umerziehung durch Arbeit auf dem Land weilten. Demnach sollten jedoch nicht alle in die Städte zurückkehren dürfen, sondern einige gezielt in andere ländliche Gebiete umgesiedelt werden und dort eine Arbeitsstelle zugeteilt bekommen. Nur diejenigen, die Verwandte in den Städten hatten, durften umgehend in die Städte zurückkehren. ${ }^{143}$

Das am 17. September 1978 ergänzend herausgegebene Dokument Nr. 55 betont in seiner Einleitung, dass die ehemaligen Rechtsabweichler ihre Stärken wieder für den Sozialismus einbringen sollen, indem ihr Enthusiasmus wiederbelebt werden solle. Zudem verweist es auf die Richtigkeit und Notwendigkeit der Durchführung der Kampagne gegen Rechtsabweichler, die demnach dazu beigetragen habe, die Diktatur des Proletariats zu gewährleisten und den Aufbau des Sozialismus sicherzustellen. Das Dokument enthält eine Reihe von weiteren Regelungen zum Umgang mit den entlassenen Rechtsabweichlern, wonach es für die Stabilität und Einheit des Landes unabdingbar sei, die Korrekturen der Einzelfälle so schnell wie möglich vorzunehmen. Die wichtigsten Punkte des Dokuments befassen sich jedoch mit den finanziellen Rahmenbedingungen rund um die Entlassung der Rechtsabweichler aus dem Laogai-System. Genau diese Regelungen sollten auch Jahre später immer wieder zu öffentlicher Kritik führen und letztlich auch einen Einfluss auf die inoffizielle Geschichtsschreibung ausüben. Demnach sollte das Gehalt derjenigen, die während der Umerziehung durch Arbeit Lohnkürzungen hinnehmen mussten, wieder heraufgestuft werden, allerdings sahen die Regelungen keine Kompensationszahlungen für die erlittenen

142 Wang 2016, S. 181.

143 Zhonggong zhongyang guanyu quanbu zhai diao youpai fenzi maozi de tongzhi 中共中央爸 于全部摘掉右派分子帽子的通知 (Mitteilung des Zentralkomitees der Kommunistischen Partei Chinas über die Entfernung aller rechten Hüte) 05.04.1978. 
finanziellen Verluste während der Jahre im Laogai-System vor. Auch die Pensionsansprüche richteten sich somit nicht nach dem vor der Umerziehung erzielten Lohn, sondern berücksichtigten lediglich den gekürzten Lohn, den die Rechtsabweichler im Laogai-System erhalten hatten. Wenn die Rechtsabweichler bereits verstorben waren, wurden zwar Pensionen an die Hinterbliebenen gezahlt, jedoch richteten sich diese ebenfalls nach dem niedrigeren Lohnniveau des Laogai-Systems. Ausnahmen sollten in Form von Hilfeleistungen nur bei besonders schweren sozialen Härtefällen gemacht werden. Die teilweise wirtschaftlich sehr negativen Folgen, die die Familien der Rechtsabweichler erdulden mussten, fanden keinerlei Berücksichtigung in den gesetzlichen Regelungen des Jahres 1978. Weiterhin besagt das Dokument, dass den Betroffenen alle konfiszierten Güter zurückzugeben seien. Ein weiterer wichtiger Punkt des Dokuments sieht vor, dass die Parteimitgliedschaft eines Rechtsabweichlers nach seiner Entlassung nicht automatisch wiederhergestellt werden würde. Damit wird deutlich, dass nicht nur keine vollständige finanzielle „Wiedergutmachung“ angestrebt worden ist, sondern dass faktisch auch keine vollständige Wiederherstellung des gesellschaftlichen Status und letztlich keine echte „Rehabilitierung“ der Rechtsabweichler durchgeführt worden ist. ${ }^{144}$ Genau genommen ist in den meisten Fällen in den Gerichtsbeschlüssen dementsprechend auch nicht von einer Rehabilitierung (pingfan 本反), sondern von der Korrektur (gaizheng 改正) eines Falles die Rede. Die in solchen Fällen in der westlichen Literatur meist verwendete Bezeichnung „Rehabilitierung“ ist in Bezug auf die sogenannten Rechtsabweichler irreführend, deshalb sollte korrekterweise die Bezeichnung „Einzelfallkorrektur“ verwendet werden. $^{145}$

Berücksichtigt werden sollte auch, dass nicht alle Fälle umgehend nach der Verabschiedung der entsprechenden Beschlüsse im Jahr 1978 juristisch aufgerollt worden sind. Für ganz China bzw. für alle zu Unrecht verurteilten Rechtsabweichler wie auch für all die anderen politischen Opfer der Mao-Zeit betrachtet, war dies ein langwieriger Vorgang, der für die meisten erst im Jahr 1980, für einige wenige Betroffene gar erst im Jahr 1984 mit ihrer Rehabilitierung bzw. Einzelfallkorrektur enden sollte. Eine Entlassung aus dem Laogai-System erfolgte in der Regel jedoch schon vor Abschluss des eigentlichen juristischen Verfahrens. ${ }^{146}$

In offiziellen Quellen werden häufig ganz andere Angaben über den Verlauf und die Anzahl von Rehabilitierungen bzw. Einzelfallkorrekturen der sogenannten Rechtsabweichler gemacht. In dem im September des Jahres 1979 herausge-

144 Zhonggong zhongyang zhuzhi bu 中共中央组织部 17.09.1978.

145 Wang 2016, S. 182.

146 Siehe Ladany 1992. 
gebenen Parteidokument Nr. 65 wurde entgegen des heutigen Forschungsstandes angegeben, dass rund ein Jahr nach der Verabschiedung der entsprechenden Beschlüsse bereits 90 Prozent der Rechtsabweichler korrigiert worden seien. ${ }^{147}$ Und in der von den Parteihistorikern Pang Xianzhi und Jin Chongji herausgegebenen offiziellen Biografie Mao Zedongs schreiben sie sogar, dass die Hüte der meisten Rechtsabweichler bereits in den Jahren 1959 bis 1964 entfernt worden seien:

Im Jahr 1959 hat das Zentralkomitee eine Direktive zur schrittweisen Entfernung der Hüte der Rechtsabweichler erlassen. Bis zum Jahre 1964 sind die meisten von ihnen, die als solche gebrandmarkt worden waren, freigesprochen worden. Im April 1979 hat das Zentralkomitee schließlich beschlossen, alle Rechtsabweichler zu rehabilitieren - sowohl die noch Lebenden als auch posthum. Im September desselben Jahres wurde festgelegt, dass die Fälle all jener neu aufgerollt werden sollten, denen Unrecht zuteilgeworden war. Bis zum Ende des Jahres 1981 sind alle Fälle korrigiert worden. ${ }^{148}$

Tatsächlich wurden ab den späten 1970er Jahren jedoch insgesamt mehrere Millionen ungerechte, falsche und fälschliche Urteile der vergangenen rund 30 Jahre aufgerollt. Alleine in Shanghai sollte in den kommenden Jahren 310.000 Opfern eine Korrektur ihres Falles oder eine Rehabilitierung zukommen. ${ }^{149}$

\section{Der Fall Gao Tingzhang}

Neben Zeitzeugenberichten ermöglicht ein Blick in Archive, den Weg der Rechtsabweichler bis zur Einzelfallkorrektur und den Kampf um eine vollständige Rehabilitierung nachzuzeichnen und nachzuvollziehen, dass eine Freilassung in der Regel eben nicht die vollständige Rückerlangung des einstigen gesellschaftlichen und materiellen Status bedeutete. Die sogenannten Rechtsabweichler hatten um viel mehr zu kämpfen, als nur um ihre Freilassung. Die Akte zur Einzelfallkorrektur des ehemaligen Rechtsabweichlers Gao Tingzhang 高庭璋, welche sich in den Archiven der Hoover Institution an der Stanford University finden lässt, zeigt beispielhaft, wie lang der Weg in die Freiheit und der meist vergebliche Kampf um eine echte „Rehabilitierung“ sein konnte. Gao Tingzhang, der in eine Familie von reichen Bauern und Grundbesitzern hineingeboren worden war, wurde am 09. September 1957 im Alter von 49 Jahren als Rechtsabweichler gebrandmarkt und schließlich am 28. Januar 1958 als Konterrevolutionär

147 Zhonggong zhongyang zhuzhi bu 中共中央组织部 10.09.1979.

148 Eigene Übersetzung. Original: Pang und Jin 2011, S. 1676.

149 Leese und Engman 2018, S. 14. 
zu 20 Jahren Haft verurteilt. Zum Zeitpunkt seiner Verurteilung arbeitete er als Bahnhofsvorsteher in Wuzhong 吴忠 (Autonome Region Ningxia). Seiner Akte lässt sich entnehmen, dass er von einem Kollegen durch ein Schreiben denunziert worden ist, welches am 08. Januar 1958 beim kommunalen Ausschuss der KPCh in Wuzhong eingegangen ist. In diesem wurde er bezichtigt, nach der Machtübernahme der Kommunisten wiederholt öffentlich reaktionäre Äußerungen getätigt zu haben. Ferner soll er geäußert haben, dass er mit der neuen politischen Führung nicht zufrieden sei, unter anderem deswegen, weil die KPCh einen tiefen Graben zwischen sich und die Massen ziehe. Außerdem besagt der Brief, dass es der Wurzel von Gao Tingzhangs Gedanken entspreche, sich gegen den Sozialismus zu stellen und von oben auf die Kommunistische Partei herabzublicken. Demnach soll er geäußert haben, dass es dem neuen China noch nicht gelungen sei, „rote Experten“ auszubilden, und dass Chinas Wissenschaft und Technologie von der Sowjetunion abhänge. Die Partei sei ein Kind, das keine führende Rolle in der Wissenschaft einnehmen könne. Außerdem seien Bürokratismus, Subjektivismus und Sektierertum erst in der neuen Gesellschaft entstanden, also durch die Herrschaft der KP ausgelöst worden - dies habe es in der alten Gesellschaft nicht gegeben. Ein weiterer Grund dafür, dass er zu Haft verurteilt wurde, lag, wie den Akten zu entnehmen ist, darin, dass er während des Bürgerkriegs der Guomindang (GMD) beigetreten sei. Gao wurde außerdem verdächtigt, ein Spion der GMD zu sein. Dies zeigt, dass Fehlurteile wie diese zur Zeit der Kampagne gegen Rechtsabweichler zu Teilen aus einem tiefen Misstrauen gegenüber ehemaligen Mitgliedern der Guomindang (GMD) rührten, insbesondere, wenn ihre ehemaligen Mitglieder Intellektuelle waren, wozu man Gao Tingzhang, der zu Beginn der 1930er Jahre Student an der Managementschule der Longhaier Eisenbahn (Provinz Fujian) gewesen ist, zweifelsfrei zählen kann. Sein Fehlurteil wurde schließlich im Jahr 1979 durch das Kreisgericht Wuzhong aufgehoben. Die Urteilsbegründung besagt, dass das ursprünglich getroffene Urteil nicht im Einklang mit der politischen Linie der Partei stehe (,Zhe shi bu fuhe dang de zhengce“这是 不符合党的政策). Dennoch ist auch Gao nicht vollständig rehabilitiert worden, und so wird in der Urteilsbegründung weder von einer Rehabilitierung noch von einer Berichtigung, sondern von einem Widerruf (chexiao 撤销) des Urteils gesprochen, was ihn gegenüber jenen, die eine Einzelfallkorrektur zugesprochen bekamen, sogar noch benachteiligte. Durch diesen Beschluss wurde erwirkt, seine im Jahre 1958 konfiszierten Gegenstände nicht an ihn zurückzugeben. Und so legte Gao Tingzhang schließlich Einspruch gegen diesen einfachen Widerruf seines Fehlurteils ein. In seinem Beschwerdeschreiben, welches am 03 . Mai 1982 beim Kreisgericht Wuzhong eingegangen ist, stellt er drei Forderungen: erstens, die Rückgabe seines konfiszierten Eigentums, insbesondere seines Goldes und der Schmuckstücke seiner Frau. In seinem Schreiben betonte er außerdem, dass er, 
als er im Jahr 1958 als Bahnhofsvorsteher in Wuzhong arbeitete, von seinem niedriger gestellten Kollegen Liu Zhenggang fälschlich denunziert worden sei, weil sich dieser, Gaos Vermutung nach, Vorteile für seine Karriere erhofft habe. Liu, so habe er später erfahren, habe auch die Konfiszierung seines Eigentums veranlasst. Deshalb forderte er, zweitens, dass man Lius Tat der Falschaussage öffentlich macht, auch wenn dieser bereits verstorben war. Drittens, forderte er die vollständige Wiederherstellung seiner Bürgerrechte („huifu le wo de gongminquan“恢复了我的公民权). Schlussendlich war er der Meinung, dass dieser einfache Widerruf seines Urteils im Widerspruch zur Verfassung der VR China stehe. Außerdem weist er in diesem Schreiben, rund 20 Jahre nach seiner Verurteilung, noch einmal alle gegen ihn im Jahr 1958 erhobenen, falschen Vorwürfe zurück, indem er erklärt, dass er zu keinem Zeitpunkt reaktionäre Äußerungen gegen die KPCh getätigt habe. Er führt aus, dass er zu Beginn der 1930er Jahre gezwungen worden sei, der Guomindang beizutreten, er aber weder Mitgliedsbeiträge gezahlt, noch Parteitreffen besucht habe. Er betont außerdem, dass er 1939 unter der Kontrolle der GMD auch mitgeholfen habe, Kommunisten zu retten. Eine wirkliche Reflexion oder Aufarbeitung des Unrechts, das ihm widerfahren ist, stellt dieser Brief zwar nicht dar, dennoch betont er in diesem auch, dass durch das Fehlurteil und die sich anschließende langjährige Haftstrafe seine Familie zerstört worden sei. Außerdem wiederholt er mehrfach, dass ihm die Gründe für seine Verurteilung jahrelang völlig unbekannt geblieben seien und er diese erst nach seiner Entlassung erfahren habe. Gao geht es in seinem Schreiben also nicht ausschließlich darum, seinen materiellen Besitz zurückzuerlangen, sondern auch darum, eine Art Gerechtigkeit zu erfahren und seinen alten Status in der Gesellschaft wiederherzustellen. Er will denjenigen, der für sein Schicksal verantwortlich war, zur Rechenschaft ziehen, auch wenn dieser im Jahr 1982 schon gar nicht mehr am Leben gewesen ist - diese Forderungen können als ein wichtiger Schritt im Prozess des Abschließens mit der Vergangenheit angesehen werden. Der Fall Gao Tingzhang zeigt auch, dass viele ehemalige Rechtsabweichler auch nach ihrer Einzelfallkorrektur noch damit haderten, dass ihnen die Gründe für ihre Verurteilung eine sehr lange Zeit unbekannt gewesen sind. Gao ist keinesfalls ein Einzelfall, auch andere ehemalige Rechtsabweichler berichten immer wieder, dass ihnen die Gründe ihrer Verurteilung sehr lange überhaupt nicht bekannt gewesen seien - manchen sind die Gründe sogar Zeit ihres Lebens nicht offengelegt worden. ${ }^{150}$ Briefe, wie die von Gao Tingzhang drei Jahre nach seiner Ein-

150 Harry Wu berichtet etwa, dass ihm die Gründe für seine Verurteilung absichtlich verschwiegen worden seien, und erinnert sich daran in seinen Memoiren wie folgt: Den oberen Abschnitt des Umerziehung-durch-Arbeit-Bescheides deckte er mit einer Hand ab und zeigte mit der anderen Hand auf die Stelle, wo ich unterschreiben sollte. Ich verlangte, er solle seine Hand weg- 
zelfallkorrektur, geben außerdem ein Zeugnis darüber, dass mit der Freilassung und der damit eingeleiteten Rückkehr in die Gesellschaft in vielen Fällen noch kein Schlussstrich gezogen worden ist und auch dass die juristische Aufarbeitung der Einzelfälle der späten 1970er bzw. frühen 1980er Jahre einigen von ihnen keine Genugtuung verschaffen konnte. ${ }^{151}$

Dies lässt sich darüber hinaus zu weiten Teilen auch darauf zurückführen, dass der Staat den Opfern keine angemessene Kompensation zukommen ließ. Tatsächlich herrschte ab den späten 1970er Jahren eine Ungleichheit im staatlichen Umgang mit den verschiedenen Opfern der „historischen Fehler“ der KPCh zwischen 1949 und 1976. Während die ehemaligen Rechtsabweichler in der Regel nur ihre zuvor konfiszierten Güter zurückerhielten, wurden den Opfern der Kulturrevolution teilweise auch Entschädigungen gezahlt, wenn auch nur in recht begrenztem Rahmen, was viele Betroffene einige Jahre später und immer wieder bis in die Gegenwart hinein als Ungerechtigkeit anprangerten. Wie die Dokumentation des Maoist Legacy Projects der Universität Freiburg zeigt, sollten die Fälle der Kampagnen vor dem Ausbruch der Kulturrevolution, also zwischen 1949 und 1966, nur politisch, aber nicht finanziell korrigiert werden. Diese Unterscheidung zwischen den verschiedenen Opfergruppen wurde durch ein am 05. Juli 1979 in Umlauf gebrachtes Parteidokument manifestiert. In diesem heißt es:

Die Fälle vor der „Kulturrevolution“ sollten in erster Linie politisch rückgängig gemacht bzw. korrigiert werden. Grundsätzlich wird es keine finanzielle Kompensation geben. In Einzelfällen, in denen eine Person nachweislich notleidend ist, sollte es eine angemessene Unterstützung in Form von Sozialhilfe durch die entsprechende Arbeitseinheit oder die zuständige Abteilung für soziale Angelegenheiten geben. ${ }^{152}$

In Bezug auf die Kampagne gegen Rechtsabweichler kann ein Grund dafür darin liegen, dass etwaige Kompensationszahlungen einem Eingeständnis gleichgekommen wären, dass der Kampf gegen die Rechten ein Fehler gewesen sei, was insbesondere zu diesem Zeitpunkt ganz und gar nicht im Sinne des Machtausbaus

nehmen: Ich wollte den Grund für meine Umerziehung sehen. Doch der Polizist sagte: „Unterschreib einfach hier.“ Worauf ich ihm entgegnete: „Ich will den oberen Teil sehen." Ein wenig erbost darüber, gab er zurück: „Wenn du unterschreiben willst, unterschreib. Wenn du ablehnst, ist es auch in Ordnung. So oder so, musst du mit mir kommen. "Wu 2009, S. 75.

151 Communist Chinese Political Movement Collection, 2014 C24.

152 Eigene Übersetzung. Original siehe: Zhonggong zhongyang, guowuyuan pizhuan caizheng bu danzu „quanguo caizheng gongzuo huiyi huibao tigang“ de tongzhi (jielu) 中共中央、国务院 批转财政部党组, 全国财政工作会议汇报提纲“的通知(节录) (Mitteilung des Zentralkomitees der KPCh und des Staatsrates über die Billigung des Entwurfs der Nationalen Finanzarbeitskonferenz der Parteigruppe des Finanzministeriums [Auszug]) 05.07.1979. 
Deng Xiaopings gewesen wäre (siehe Kap. 4). Am wahrscheinlichsten ist jedoch, dass sich der Hauptgrund für die Ungleichbehandlung der Opfer der Kulturrevolution und den vorangegangenen Kampagnen vorwiegend auf die finanzielle Situation des chinesischen Staates zurückführen lässt. Nach dem Ende der Kulturrevolution befand sich die chinesische Wirtschaft in einer sehr schwierigen Lage. Die Readjustierung der Wirtschaft hatte Priorität, sodass die Erben Maos nicht Willens gewesen sein dürften, für den wirtschaftlichen Aufbau des Landes benötigte finanzielle Mittel für eine angemessene Kompensation der Opfer aufzubringen. ${ }^{153}$

Für die Betroffenen gestaltete sich die Rückkehr in die Gesellschaft nach dem Ende des Mao-Zeitalters jedoch nicht nur aufgrund der mangelnden finanziellen Kompensation schwierig, sondern vor allem deshalb, weil Täter und Opfer Seite an Seite leben und gemeinsam das Land aufbauen mussten. Viele ehemalige Rechtsabweichler waren, ähnlich wie viele Opfer der Kulturrevolution, von Nachbarn, Kollegen oder Kommilitonen angeschwärzt und deshalb erst verurteilt worden. Familien und Freundschaften sind zerbrochen und es herrschte noch immer ein tiefes Misstrauen gegenüber jenen, die jahrelang als politische Feinde gebrandmarkt worden waren. Und somit lässt sich festhalten, dass, auch wenn die meisten der als Rechtsabweichler Gebrandmarkten nach dem Tode Maos formal schnell wieder ihre Freiheit zurückerlangt hatten, der Weg der gesellschaftlichen Rehabilitierung noch lange nicht abgeschlossen war. Die Reflexion der Vergangenheit durch das Teilen von persönlichen Erinnerungen, zum Beispiel in Form von Memoiren oder durch andere Formen der inoffiziellen Geschichtsschreibung, stellte einen weiteren wichtigen Schritt der persönlichen „Rehabilitierung“ der ehemaligen Rechtsabweichler dar.

153 Zur Readjustierung der chinesischen Wirtschaft nach dem Beginn der Reform- und Öffnungspolitik siehe Brodsgaard 1983, S. 257 ff. 